\title{
Role of Vasopressin in the management of Atonic Postpartum Hemorrhage.
}

\author{
Dr T N Vasudeva Panicker \\ DA MD DGO, Anesthesiologist and Gynecologist Consultant Panicker'sHospital Kodungallur Thrissur Kerala \\ India.Email:_drvpanicker@gmail.com Website:drpanicker.com
}

\begin{abstract}
Intra-myometrial Vasopressin has been studied in 24 patients of atonic PPH intractable to uterotonics (oxytocin and methergin) and PGF2alpha. 20 units of vasopressin diluted in $100 \mathrm{ml}$ of normal saline was infiltrated intra myometrially $(n=7)$ at multiple sites using $23 G$ spinal needle under direct vision at $C S$, and trans-abdominally $(n=17)$ in case of normal vaginal delivery. Atonic PPH was controlled in all women with absolute success. Intra myometrial Vasopressin showed immediate response with complete hemostasis and uterine tonicity within 4 to 8 minutes in our study.
\end{abstract}

Key words: Vasopressin, Atonic PPH (postpartum Hemorrhage), Intramyometrium

\section{Introduction}

Postpartum hemorrhage is the single major cause of maternal mortality worldwide. More than 100 thousand maternal deaths occur every year globally due to PPH alone. Postpartum hemorrhage is a common obstetric emergency which cannot be predicted by any means even today. How ever many of the deaths can be prevented if certain simple measures and precautions are considered. Unpredictable sudden massive bleeding occurring after delivery in a difficult situation like low resource settings results in maternal death. When simpler measures like uterotonics, uterine massage, uterine packing, bimanual compression, and balloon tamponade are not successful, the women has to be shifted to higher medical centers for further techniques like B-Lynch suturing, stepwise devascularization and internal iliac ligation, angioembolisation to stop bleeding. Most of the times mothers die in transit or reach in a state of extreme shock at higher medical centers. In developing countries with high prevalence of anemia PPH proves disastrous. A minimal blood loss when there is hemodynamic compromise is very significant and life threatening. There is every need for simpler and safer techniques, which can be practiced with minimal expertise even in low resource settings to treat PPH.

The key to control of PPH and prevention of mortality lies on early initiation of therapeutic measures as soon a diagnosis of PPH is made .Prompt action ,safety, efficacy, easy administration is requisite for an ideal drug or method of choice for atonic PPH. In this study we have used dilute vasopressin intra myometrially infiltrated at different sites (also transabdominaly) as an immediate preliminary method to control atonic PPH which did not respond to uterotonics. Vasopressin infiltration is simple procedure which takes only a few minutes like any intramuscular injection.

Vasopressin was synthesized in 1952. It is extensively used in ICU for vasodilatory shock and cardiac arrest. It is used in diabetes insipidus, and bleeding esophageal varices. Vasopressin is also being used widely in gynecological surgeries like myomectomy (1) vaginal hysterectomy, abdominal hysterectomy, ovarian cystectomy, and hysteroscopy (2). Sub endometrial injection of vasopressin was reported for the management of placenta praevia (3), and in second trimester dilatation and Curettage (4) to reduce bleeding by different authors. Lurie et al had reported about the use of local vasopressin for control of PPH in Placenta accereta. Extensive literature search revealed the following rare complications after vasopressin infiltration that include pulmonary edema, severe hypotension, myocardial infarction, bradycardia (5-7) which could be due to accidental intravascular injection.

\section{Materials \& Methods:}

When medical management failed to control bleeding in atonic PPH, 20 units of vasopressin diluted in $100 \mathrm{ml}$ of normal saline was infiltrated intra myometrially at multiple sites using $23 \mathrm{G}$ spinal needle under direct vision at CS, and trans-abdominally in case of normal vaginal delivery. Uterus was lifted up and forwards bimanually and stabilized by an assistant to avoid bowel injury. Method of administration is shown in the video link https://www.youtube.com/watch? $=5 \mathrm{CbYMbWpxQY}$. And Figure 1 and 2.The study confirms to standards of declaration of Helsinki. 


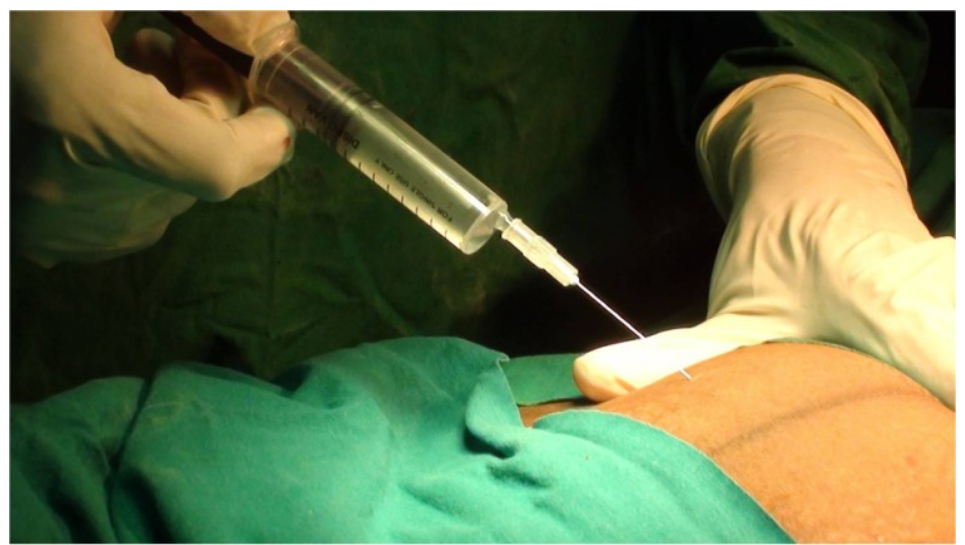

Figure 1.Transabdomial intramyometrial Vasopressin Administration.

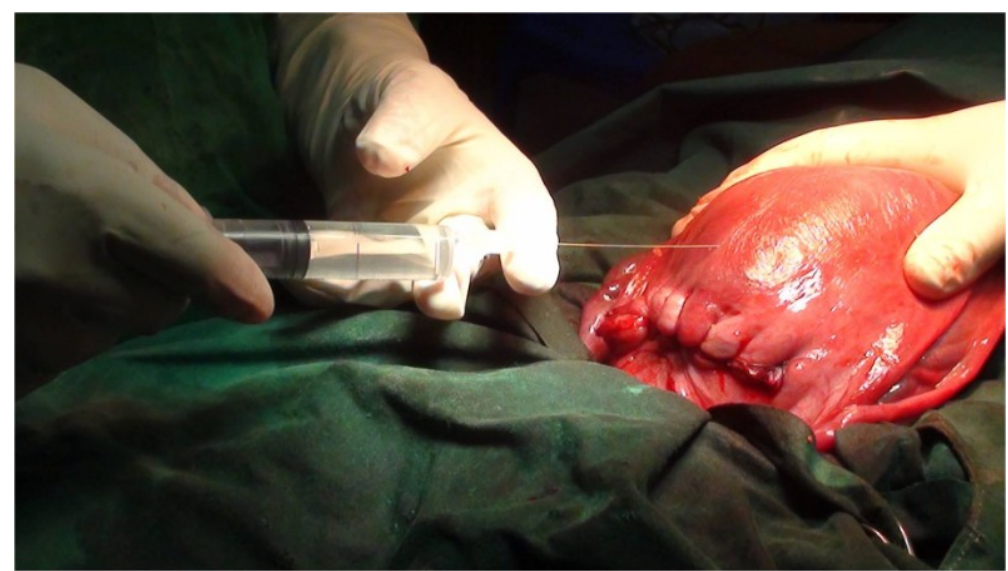

Figure 2. Direct Intramyometrial administration of diluted Vasopressin for Atonic PPH.

Vital signs were monitored during the procedure. Always aspirate for blood during intra myometrial infiltration to avoid intra vascular injection.

\section{Results:}

The study was carried out for 4 years during which 4400 deliveries were recorded in our institution. In 92 women excessive bleeding was observed following delivery. Routine measures like uterine massage, intravenous methergine and 20U oxytocin infusion and prostaglandin PGF2 alpha (IM 2doses (500 microgram) was given. In 24 women bleeding could not be controlled with the above treatment measures, and the atonic PPH continued. All these 24 women were treated with intra myometrial infiltration of vasopressin. A blanching like effect was observed on myometrium at the site of infiltration as shown in Figure 3 .On an average the $100 \mathrm{ml}$ of the diluted vasopressin can be administered with three to four punctures over the uterus. The results are displayed in the table 1. There was no major adverse events and mortality in our series. In four of 24 women PGF 2alpha was contra indicated due to severe asthma. One of these four women developed atonic PPH after two hours of caesarean delivery, when she was getting nebulized with salbutamol.

Table: 1

\begin{tabular}{|c|l|c|}
\hline \multicolumn{1}{|c|}{ Characteristics } & \multicolumn{1}{|c|}{ Number } \\
\hline 1 & Parity & 42 \\
& Primigravida & 50 \\
\hline 2 & Multigravida & 12 \\
& Medical disorders in pregnancy & 2 \\
& Hypertensive disorders & 4 \\
& Gestational diabetes mellitus & 12 \\
\hline 3 & Bronchial Asthma & 59 \\
& Anemia & 15 \\
& Mode of delivery & 18 \\
\hline & Spontaneous vaginal delivery & 68 \\
\hline
\end{tabular}


Role of Vasopressin in the management of Atonic Postpartum Hemorrhage.

\begin{tabular}{|c|l|c|}
\hline & Postpartum blood transfusions & 03 \\
\hline 5 & Vasopressin used & 24 \\
& Total & 2 \\
& Postpartum blood transfusions & 07 \\
& Direct intramyometrial injection & 17 \\
& Transabdominal injection & \\
\hline & Total Cases & 92 \\
\hline
\end{tabular}

In two women secondary PPH occurred 14 days after caesarean delivery. Bleeding did not stop with routine measures. Ultra sound scanning showed grossly enlarged uterus with wide endometrial cavity filled with blood clots. Twenty units of vasopressin in $100 \mathrm{ml}$ saline were infiltrated in to the myometrium trans abdominally. Bleeding stopped within 10minutes. Next day repeat scan showed well contracted uterus with thin endometrial cavity. Increase in BP was observed in 4 women, which responded well for nitroglycerin infusion. (25mg in $500 \mathrm{ml}$ normal saline - rate of infusion adjusted according to the response).Two of these women had PIH which was well controlled at the time administration of Vasopressin.

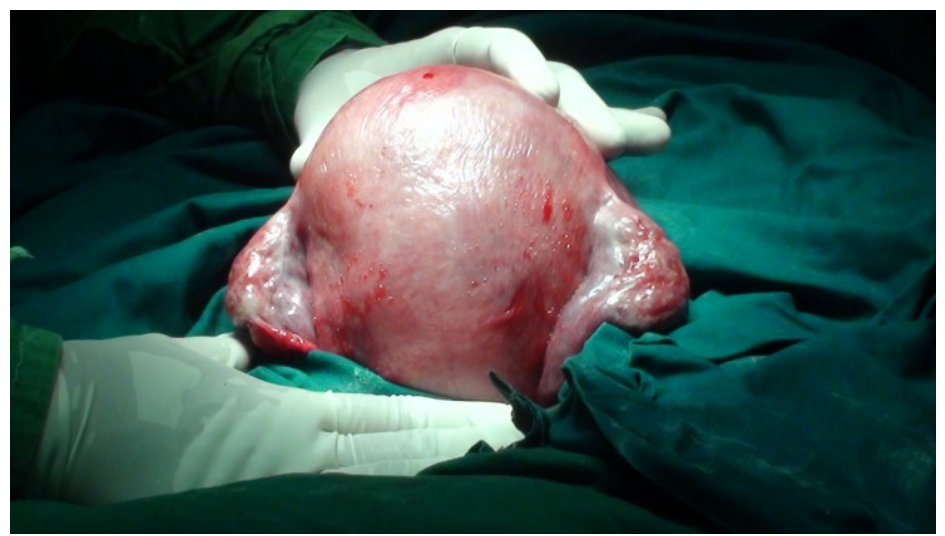

Figure 3. The blanching effect due to profound vasoconstriction and tonicity of uterus noted after administration of intramyometrial Vasopressin.

\section{Discussion}

Bleeding after parturition is mainly controlled by 3 mechanisms. Myometrial contraction and retraction, clot formation and plugging of open sinuses at the placental bed, and opposition of uterine walls. All uterotonic drugs acts by myometrial contraction only. But vasopressin stops PPH by (1) Myometrial contraction by action on oxytocin type receptors. (2) Vasoconstriction by increased release of intra cellular $\mathrm{Ca}+$ and blockade of ATP sensitive $\mathrm{K}+$ channels in the vascular smooth muscles - V1 receptor action. (3) By liberation of factor VIII and Von Willebrand factor and also by platelet activation at vascular endothelial cells resulting in increased clot formation - V2 receptor effect.

Vasopressin causes intense vasospasm at the site of injection, resulting in reduced absorption and systemic dissemination. However, sometimes it can produce side effects like bradycardia and acute hypertension, could be due to accidental intravascular injection. Severe bradycardia can occur in women under spinal anesthesia due to unopposed vagal activity which results due blockade of cardiac sympathetic fibers. For the same reason protective reflex tachycardia in response to hypotension fails. Hence atropine should be given immediately if bradycardia begins.

In our series bleeding could be stopped in all women with atonic PPH with intra myometrial infiltration of vasopressin within 4 to 8 minutes and none of them required surgical intervention. We did not encounter any serious side effects with this technique. Vasopressin infiltration is a cost effective technique which require minimal skills, and should find a place in all PPH kits. Further randomized trials with larger sample size will prove the efficacy of vasopressin as drug of choice for intractable PPH.

\section{Conclusion}

Intramyometrial Vasopressin showed immediate response with absolute success in all the cases of our study. This simple Life saving measure should be considered for both prophylactic and therapeutic benefits. We recommend Intra myometrial Vasopressin in the armamentarium of management of atonic PPH. 


\section{References}

[1]. Frederick J, Fletcher H, Simeon D, Mullings A, Hardie M. - Intramyometrial vasopressin: AA haemostatic agent during myomectomy. Br J Obstetric Gynaecol 1944;101:435-7

[2]. Corson SL, Brooks PG, Serden SP, Batzer FR, Gocial B. Effects of vasopressin administration during hysteroscopic surgery. J Reprod Med 1994;39:419-23

[3]. Lurie S, Appleman Z, Katz Z. Sub endometrial vasopressin to control intractable placental bleeding. Lancet 1997;349:698-9

[4]. Schulz KF, Grimes DA, Christensen DD. Vasopressin reduces blood loss from second-trimester dilation and evacuation abortion. Lancet 1985; 17:353-6

[5]. Tulandi T, Beique F, Kimia M. Pulmonary edema: A complication of local injection of vasopressin at laparoscopy. Fertil Steril $1996 ; 66: 478-80$

[6]. Nezhat F, Admon D, Nezhat CH, Dicorpo JE, Nezhat C. Life threatening hypotension after vasopressin injection during operative laparoscopy, followed by uneventful repeat laparoscopy. J Am Assoc Gynecol Laparosc 1994;2:83-6

[7]. Martin JD, Shenk LG, Intraoperative myocardial infarction after paracervical vasopressin infiltration. Anesth Analg 1994;79:1201- 